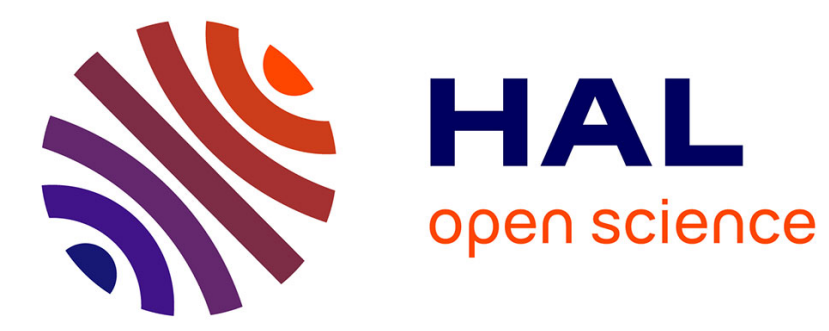

\title{
Diffusion de l'arsenic dans les structures silicium-sur-isolant obtenues par implantation d'oxygène
}

N. Guillemot, P. Normand, D. Tsoukalas, P. Chenevier

\section{To cite this version:}

N. Guillemot, P. Normand, D. Tsoukalas, P. Chenevier. Diffusion de l'arsenic dans les structures silicium-sur-isolant obtenues par implantation d'oxygène. Revue de Physique Appliquée, 1988, 23 (8), pp.1369-1373. 10.1051/rphysap:019880023080136900 . jpa-00245955

\section{HAL Id: jpa-00245955 https://hal.science/jpa-00245955}

Submitted on 1 Jan 1988

HAL is a multi-disciplinary open access archive for the deposit and dissemination of scientific research documents, whether they are published or not. The documents may come from teaching and research institutions in France or abroad, or from public or private research centers.
L'archive ouverte pluridisciplinaire HAL, est destinée au dépôt et à la diffusion de documents scientifiques de niveau recherche, publiés ou non, émanant des établissements d'enseignement et de recherche français ou étrangers, des laboratoires publics ou privés. 


\title{
Diffusion de l'arsenic dans les structures silicium-sur-isolant obtenues par implantation d'oxygène
}

\author{
N. Guillemot $\left({ }^{1}\right)$, P. Normand $\left({ }^{2}\right)$, D. Tsoukalas $\left({ }^{2}\right)$ et P. Chenevier $\left({ }^{1}\right)$ \\ ${ }^{1}$ ) Laboratoire de Physique des Composants à Semiconducteurs, UA-CNRS 840, ENSERG, 23 av. des \\ Martyrs, 38031 Grenoble, France \\ ${ }^{2}$ ) Institute of Microelectronics, Democritos Research Center for Physical Sciences, 15310 Aghia Paraskevi, \\ Attiki, Athens, Greece
}

(Reçu le 25 mars 1988, accepté le 25 avril 1988)

\begin{abstract}
Résumé. - Dans cet article, les auteurs étudient la diffusion de l'arsenic dans les structures Silicium-SurIsolant obtenues par implantation d'oxygène (SIMOX). Malgré la bonne qualité du matériau, analysé par microscopie électronique sur tranche (XTEM), les mesures par spectroscopie de masse d'ions secondaires (SIMS) et par Spreading Resistance (SR) montrent que l'arsenic est accéléré et que des impuretés inactives s'accumulent dans la partie supérieure du film de silicium. Une explication de ces deux phénomènes est proposée, puis le modèle de diffusion avec piégeage développé par McNabb et Foster est utilisé pour simuler ces effets.

Abstract. - In this article, the arsenic diffusion in Silicon-On-Insulator structures formed by oxygen implantation (SIMOX) is studied. Though cross-sectional transmission electron microscopy (XTEM) analysis confirms the high quality of the material, secondary ion mass spectroscopy (SIMS) and Spreading Resistance measurements (SR) show a pile-up phenomenon as well as an enhanced diffusivity of arsenic in the silicon overlayer. An explanation of these two observations is proposed and the McNabb and Foster equations for diffusion with trapping are solved in order to simulate these effects.
\end{abstract}

\section{Introduction.}

Parmi toutes les structures silicium-sur-isolant, le SIMOX (Separation by IMplanted Oxygen) [1]), où l'isolant est un oxyde enterré obtenu par implantation d'oxygène, semble être le matériau le plus prometteur [2]. Les recuits à haute température [3, 4] (HTA), mis au point par Stoemenos et al., ont permis d'obtenir un film de silicium monocristallin d'excellente qualité possédant une interface très abrupte vis-à-vis de l'oxyde enterré, et où pratiquement tous les précipités d'oxyde se sont dissous. Seules subsistent des dislocations qui prennent naissance à l'interface $\mathrm{Si} / \mathrm{SiO}_{2}$ pour disparaître à la surface du silicium (dislocations dites traversantes), peu gênantes pour la fabrication de dispositifs.

La diffusion des dopants dans cette structure a été très peu étudiée [5], sans doute parce que le procédé de fabrication du matériau était jusqu'à présent en constante évolution. Avec les recuits HTA, on peut raisonnablement penser que la structure est pratique- ment stabilisée [6]. Il devient alors nécessaire d'étudier la redistribution des impuretés dans le film de silicium lors des recuits thermiques si l'on veut maîtriser parfaitement la fabrication des circuits intégrés silicium.

Dans cet article les auteurs étudient la diffusion de l'arsenic implanté dans une structure SIMOX dont la bonne qualité est vérifiée par microscopie électronique sur tranche (XTEM). Le profil du dopant après des recuits à différentes températures est caractérisé par spectroscopie de masse d'ions secondaires (SIMS) et par Spreading Resistance (SR). Ces profils sont d'autre part simulés avec les modèles de diffusion classiquement employés sur silicium massif [7] ; la comparaison avec l'expérience montre une accélération de la diffusion dans la moitié supérieure du silicium, ainsi qu'une accumulation d'impuretés électriquement inactives à la surface. Une explication de ces phénomènes est proposée puis le modèle de diffusion avec piégeage développé par $\mathrm{McNabb}$ et Foster [8] est utilisé pour simuler ces effets. 


\section{Expériences.}

Le substrat de SIMOX, dont nous disposons, a été fabriqué au LETI/CEA Grenoble par implantation à $200 \mathrm{keV}$ et $600{ }^{\circ} \mathrm{C}$ d'une dose de $1,5 \times 10^{18} \mathrm{~cm}^{-2}$ $\mathrm{d}^{\prime} \mathrm{O}^{+}$dans une plaque de silicium de type $\mathrm{P}$ et d'orientation $\langle 100\rangle$. La plaque, encapsulée par un oxyde déposé, a été recuite pendant 6 heures sous argon à haute température $\left(1320^{\circ} \mathrm{C}\right)$, afin d'obtenir un film de silicium de très bonne qualité au-dessus de l'oxyde enterré $[3,4]$. L'épaisseur de cette fine couche de silicium $(0,22 \mu \mathrm{m})$ a été mesurée optiquement (mesure en réflectivité par spectroscopie) [9].

Une fois l'oxyde protecteur enlevé, la plaque est implantée à l'arsenic $\left(\mathrm{As}^{+}, 120 \mathrm{keV}, 10^{15} \mathrm{~cm}^{-2}\right)$. L'énergie est choisie pour que le maximum de concentration se situe à peu près au milieu de la couche de silicium [10] et la dose est suffisamment faible pour qu'il n'y ait pas formation d'agglomérats (clusters) lors des recuits ultérieurs, comme il a été observé sur silicium massif [11-13]. Pour éviter toute contamination dans les fours, un nouvel oxyde de $0,75 \mu \mathrm{m}$ d'épaisseur est déposé et 2 échantillons de cette plaque sont recuits sous azote, le premier à $900{ }^{\circ} \mathrm{C}$ pendant $100 \mathrm{mn}$, le second à $950^{\circ} \mathrm{C}$ pendant $60 \mathrm{mn}$.

\section{Mesures et résultats.}

Une analyse de nos échantillons par microscopie électronique sur tranche (XTEM) confirme l'excellente qualité cristalline du silicium, malgré des dislocations traversantes, tous les $0,2 \mu \mathrm{m}$ environ

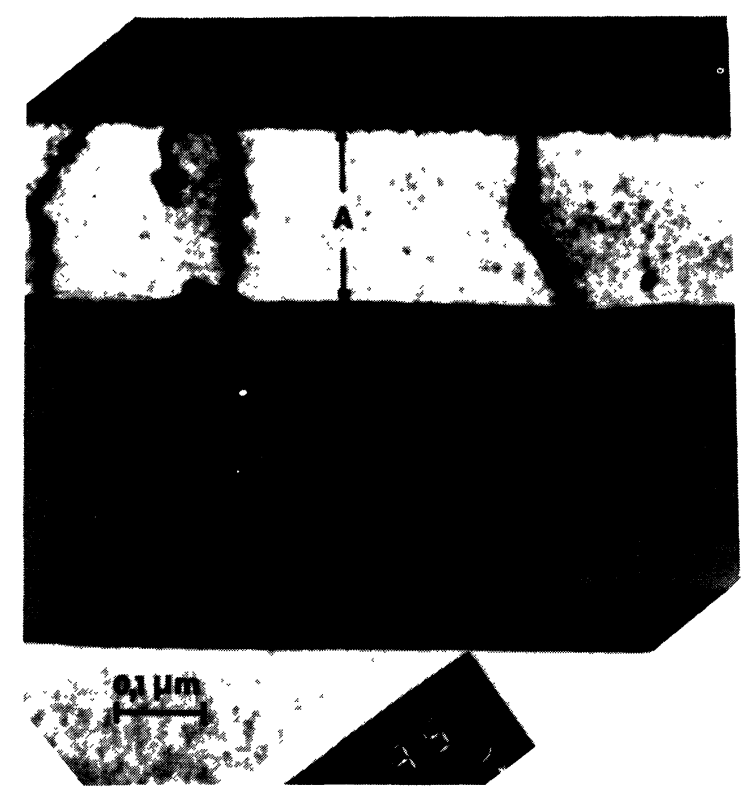

Fig. 1. - Vue en coupe du matériau SIMOX par microscopie électronique sur tranche: A représente le film de silicium et B l'oxyde enterré.

[Cross-sectional micrograph of the SIMOX structure : A denotes the silicon film and B the buried oxide.]
(Fig. 1). Notre plaque de SIMOX a été fabriquée en 1986; il faut noter qu'avec deux implantations successives d'oxygène, Margail et al. obtiennent actuellement des structures SIMOX de qualité encore supérieure [14].

Le profil chimique d'arsenic immédiatement après implantation est observé dans la couche de silicium et dans l'oxyde enterré par spectroscopie de masse d'ions secondaires (SIMS) afin d'ajuster dans les simulations les paramètres d'implantation. La redistribution du dopant pendant les recuits thermiques est étudiée par SIMS et Spreading Resistance (SR). Ces résultats sont ensuite simulés avec le modèle développé par Fair à partir d'études sur silicium massif [7] en faisant l'hypothèse, très raisonnable, que les oxydes enterrés et déposés se comportaient comme des barrières à la diffusion [15] (flux de diffusion nul aux interfaces). Ce modèle, utilisé dans de nombreux programmes de simulation, dont le plus connu est certainement SUPREM [16], donne d'excellents résultats sur silicium massif mais dans notre structure il faut noter 2 phénomènes importants (Fig. 2) :

1) La présence d'une accumulation d'impuretés vers l'interface silicium/oxyde déposé ;

2) une accélération de la diffusion sur la première moitié du silicium.

Les impuretés accumulées sont inactives : les mesures de Spreading Resistance ne montrent aucune chute de la résistivité dans la région du pic (Fig. 3).

Des phénomènes d'accumulation de dopants ont déjà été observés sur silicium massif pour le phosphore $[17,18]$, l'antimoine et l'arsenic $[17,19]$, à l'interface oxyde/silicium lors de recuits inertes précédés d'un recuit oxydant, l'interface se comportant en quelque sorte comme un piège pour les impuretés. Dans notre cas, cette accumulation est très probablement due à la présence d'oxygène et de contaminants (chrome, azote et carbone) qui se redistribuent vers la surface du silicium après l'implantation d'oxygène, lors du recuit à très haute température, phénomène souvent observé dans les structures $\operatorname{SIMOX}[6,20$, 21]. Des complexes arsenic-oxygène et arseniccontaminant se formeraient dans cette zone, qui se comporterait alors comme un puits pour les impuretés. Le modèle de diffusion des impuretés avec piégeage proposé par McNabb et Foster [8] est appliqué par la suite pour simuler ce phénomène.

Comme il a été observé sur le silicium massif [2225], l'accélération de la diffusion de l'arsenic dans la première moitié du silicium peut s'expliquer par les défauts (auto-interstitiels) créés pendant l'implantation du dopant dans la partie amorphisée du semiconducteur [26]. On peut considérer, en première approximation, que, dans cette partie du silicium, la diffusivité de l'arsenic est proportionnelle au nombre de défauts, or il a été montré [27] que l'oxyde en 


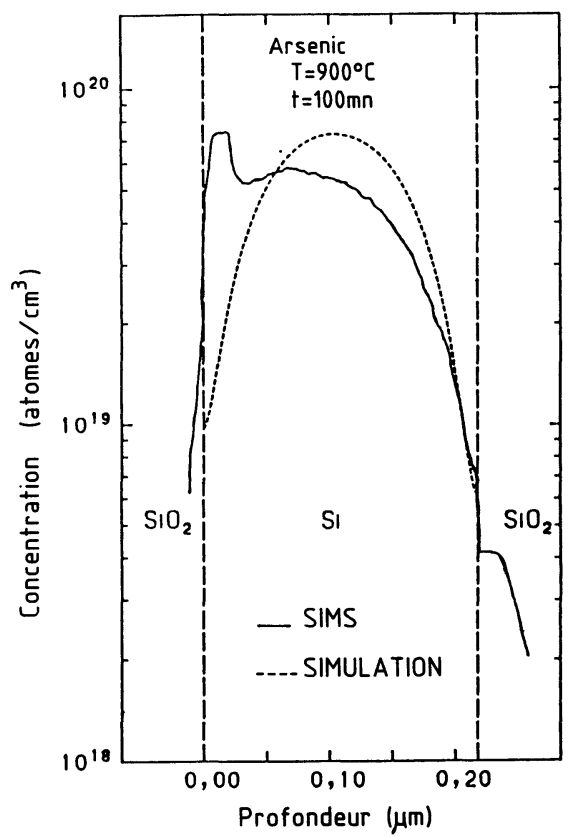

a)

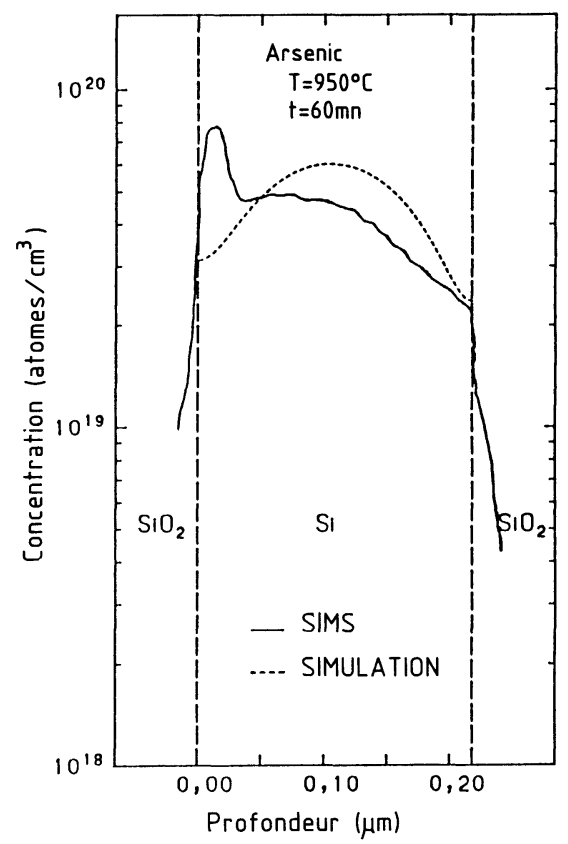

b)

Fig. 2. - Profils d'arsenic mesurés par SIMS et comparés avec la simulation (modèle proposé par Fair [7]) : (a) après un recuit à $900^{\circ} \mathrm{C}$ pendant $100 \mathrm{mn}$ (échantillon 1 ) ; (b) après un recuit à $950^{\circ} \mathrm{C}$ pendant $60 \mathrm{mn}$ (échantillon 2).

[Experimental (SIMS) and simulated (Fair's model [7]) profiles of arsenic: (a) annealed for $100 \mathrm{mn}$ at $900^{\circ} \mathrm{C}$ sample 1 ; (b) annealed for $60 \mathrm{mn}$ at $950{ }^{\circ} \mathrm{C}$ sample 2.]

surface se comportait comme un puits pour ces défauts, qui sont en quelque sorte pompés vers l'interface. En parfaite concordance avec ces observations, nous montrerons par la suite que la diffusi-

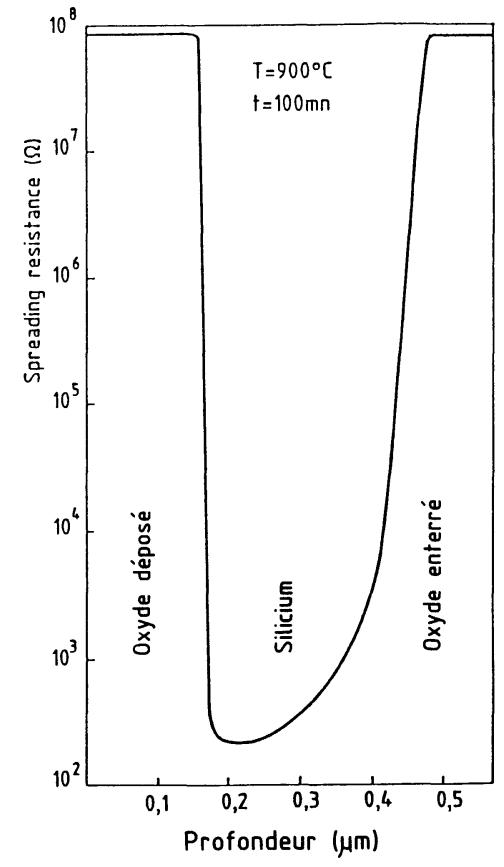

a)

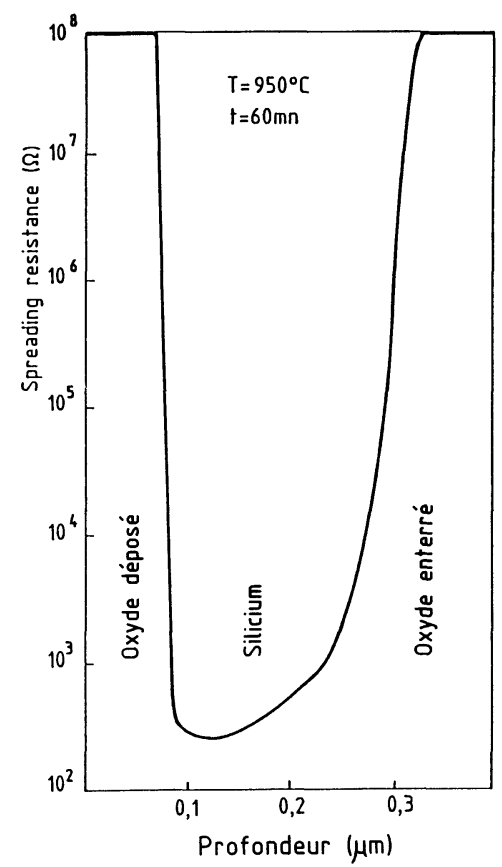

b)

Fig. 3. - Mesures de Spreading Resistance sur (a) l'échantillon 1, (b) l'échantillon 2. Le pas de mesure est de $70 \AA$ pour l'échantillon 1 et de $50 \AA \AA$ pour l'échantillon 2 .

[Spreading Resistance profiles of (a) sample 1, (b) sample 2. Measurement steps : $70 \AA$ for sample 1 and $50 \AA$ for sample 2.]

vité de l'arsenic est maximale à l'interface silicium/oxyde déposé et qu'elle décroît pour atteindre, au milieu du film de silicium, des valeurs comparables à celles obtenues sur silicium massif. 


\section{Simulation.}

Dans cette partie, nous nous proposons de simuler le phénomène d'accumulation à l'interface silicium/(oxyde déposé) avec le modèle développé par $\mathrm{McNabb}$ et Foster [8]. Les complexes arsenic-oxygène et arsenic-contaminant qui se forment dans cette zone agissent comme des pièges capturant et relâchant les impuretés suivant une loi d'action de masse. Avec l'hypothèse d'une cinétique du premier ordre, si $P(x)$ est le nombre de pièges localisés à la profondeur $x$ (où $x=0$ correspond à l'interface silicium/(oxyde déposé)), et $\phi(x, t)$ la fraction de pièges occupés en $x$ au temps $t$, la concentration totale en impuretés $C_{\mathrm{t}}(x, t)$ peut s'écrire :

$$
C_{\mathrm{t}}(x, t)=C(x, t)+\phi(x, t) P(x)
$$

où $C(x, t)$ est la concentration en atomes diffusants.

En première approximation, on suppose une concentration en pièges constante dans la couche supérieure du film de silicium $(0 \leqslant x \leqslant 0,02 \mu \mathrm{m})$, soit $P(x)=P$, et nulle en profondeur $(x>0,02 \mu \mathrm{m})$. L'équation de la diffusion modifiée qui gouverne la redistribution de l'arsenic s'écrit alors comme suit :

$$
\begin{aligned}
\frac{\partial C(x, t)}{\partial t}+P(x) \frac{\partial \phi(x, t)}{\partial t} & = \\
=\frac{\partial}{\partial x} & {\left[D(C) \frac{\partial C(x, t)}{\partial x}\right] }
\end{aligned}
$$

avec

$$
\frac{\partial \phi(x, t)}{\partial t}=k C(x, t)[1-\phi(x, t)]-k^{\prime} \phi(x, t)
$$

Le premier terme $(k C(x, t)[1-\phi(x, t)])$ représente la vitesse de capture des impuretés alors que le second terme $\left(k^{\prime} \phi(x, t)\right)$ correspond à la vitesse de dépiégeage. Il faut remarquer que lorsque $k^{\prime}=0$, les équations précédentes décrivent un piégeage irréversible. Le système d'équations est résolu sur le domaine de silicium par une méthode de différences finies [28], en utilisant un schéma de Crank-Nicholson [29].

Le piégeage n'accélère pas sensiblement la diffusion des impuretés sauf au voisinage immédiat des pièges. Dans notre cas il a été nécessaire de modifier le coefficient de diffusion effectif proposé par Fair [7]. L'accélération de la diffusion sur la première moitié du silicium a été simulée en faisant croître linéairement, à partir de $x=0,1 \mu \mathrm{m}$, ce coefficient jusqu'à un facteur 12 en $x=0 \mu \mathrm{m}$.

Sur la figure 4 sont présentés les résultats de la simulation, en excellent accord avec les profils expérimentaux. La simulation montre un piégeage très rapide des dopants, ce qui explique pourquoi la dose d'impuretés inactives et piégées est sensiblement la même à la fin de chaque recuit ( $4 \%$ et $3 \%$ )

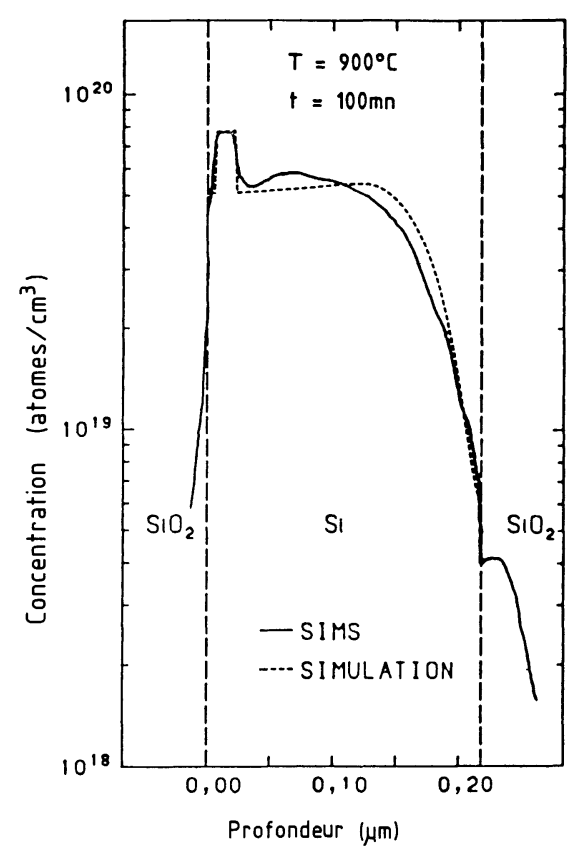

a)

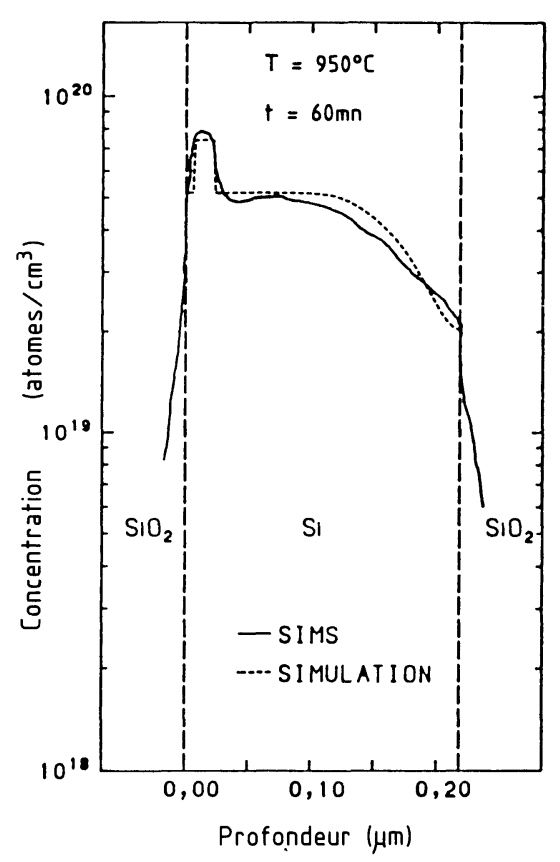

b)

Fig. 4. - Profils d'arsenic mesurés par SIMS sur (a) l'échantillon 1, (b) l'échantillon 2 et comparés avec la simulation (notre modèle).

[Comparison between SIMS and simulated (our model) profiles of arsenic for (a) sample 1, (b) sample 2.]

de la dose implantée à $900{ }^{\circ} \mathrm{C}$ et $950^{\circ} \mathrm{C}$ respectivement).

Si les pièges sont dus à la formation de complexes avec les contaminants, leur nombre est indépendant 
de la dose d'impuretés implantées. Le modèle permet alors de prévoir la proportion d'impuretés piégées, qui sera d'autant plus importante que la concentration totale est faible; aux fortes doses $\left(>5 \times 10^{15} \mathrm{~cm}^{-2}\right)$ il est très probable que cet effet disparaisse complètement.

\section{Conclusion.}

Dans cet article nous étudions la diffusion de l'arsenic dans une structure SIMOX avec des mesures de Sonde Ionique et Spreading Resistance. Une accumulation d'impuretés inactives apparaît à la surface du film de silicium, dans une zone où de l'oxygène et des contaminants se redistribuent lors de l'implantation d'oxygène, formant des complexes avec l'arsenic. Une accélération du dopant est également observée dans la première moitié du silicium, ce phénomène est très probablement dû aux interstitiels générés lors de l'implantation d'arsenic dans la partie amorphisée du film de silicium. Nous avons simulé très correctement ces effets en introduisant un piégeage (modèle proposé par McNabb et Foster), et une accélération de la diffusion dépendant de la profondeur.

\section{Remerciements.}

Les auteurs tiennent à remercier Dr. C. Jaussaud et Dr. J. Margail pour les substrats SIMOX, C. Pudda pour l'implantation d'arsenic et B. Blanchard pour les mesures SIMS (tous membres du LETI/CEA Grenoble). Ils ont eu de fructueuses discussions avec Dr. E. Scheid (ENSERG/INP Grenoble), ainsi qu'avec Pr. J. Stoemenos (Université de Tessalonique, Grèce), qui a réalisé les mesures au TEM.

\section{Bibliographie}

[1] Izumi, K., Doken, M. and ARIYoshi, H., Electron. Lett. 14 (1978) 593.

[2] Partridge, S. L., Solid State Devices 1985, Eds. P. Balk and O. G. Folgerth (Elsevier Science Publishers B.V., Printed in the Netherlands) 1986.

[3] Stoemenos, J., Jaussaud, C., Bruel, M. and MARgail, J., J. Cryst. Growth 73 (1985) 5546.

[4] Jaussaud, C., Stoemenos, J., Margail, J., DupuY, M., BlanchaRd, B. and BRUEl, M., Appl. Phys. Lett. 46 (1985) 1064.

[5] Fahey, P. and Solmi, S., J. Appl. Phys. 60 (1986) 4329.

[6] Davis, J. R., Reeson, K. and Hemment, P. L. F. Proc. ESSDERC'87 Conf. (Bologna, Italy) 1987.

[7] FAIR, R. B., Impurity Doping Process in Silicon, Ed. F.F.Y. Wang (North Holland) 1981.

[8] MCNABb, A. and Foster, P. K., Trans. TMS-AIME 227 (1963) 618.

[9] Margail, J., Thèse INP Grenoble (1987).

[10] Gibbons, J. F., Johnsons, W. S. and Mylroie, S. W., Projected Range Statistics, Stroudsburg, PA (Dowdon, Hutchinson et Ross) 1975.

[11] Tsai, M. Y., et al., J. Appl. Phys. 51 (1980) 3230.

[12] Fair, R. B. and Weber, G. R., J. Appl. Phys. 24 (1973) 272.

[13] Guerrero E., et al., J. Electrochem. Soc. 129 (1982) 1826.

[14] Margail, J., Stoemenos, J., Jaussaud, C. et Bruel, M., Proc. European Silicon On Insulator Workshop (Meylan, France) 1988.
[15] Douglas, E. C. and Dingwall, A. G. F., IEEE Trans. Electron. Devices ED-21 (1974) 324.

[16] Ho, C. P., Plummer, J. D., Hansen, S. E. and DuTTON, R. W., IEEE Trans. Electron. Devices ED-30 (1983) 1438.

[17] SaKamoto, K., Nishi, K., ICHIKawa, F. and Ushio, S., J. Appl. Phys. 61 (1987) 1553.

[18] Schwartz, S. A., Barton, R. W., Ho, C. P. and Helms, C. R., J. Electrochemic. Soc., 128 (1981) 1101.

[19] Sai-Halasz, G. A., Short, K. T. and Williams, J. S., IEEE Electron. Devices Lett. EDL-6, no 6, (1985).

[20] Kilner, J. A. (Communication privée).

[21] Reeson, K. J., et al., Proc. European Silicon On Insulator Workshop (Meylan, France) 1988.

[22] Car, R., Kelly, P. J., Oshiy ama, A. and PanteliDES, S. T., Phys. Rev. Lett. 54 (1985) 360.

[23] Angelucci, R., Negrini, P. et Solmi, S., Appl. Phys. Lett. 49 (1986) 1468.

[24] Servidori, M., Angelucci, R., Cemballi, F., Negrini, P. et Solmi, S., J. Appl. Phys. 61 (1987) 1834.

[25] Bronner, G. B. and Plummer, J. D., Appl. Phys. Lett. 46 (1985) 510.

[26] Pinizzoto, R. F., J. Cryst. Growth, 63 (1983) 559.

[27] Hu, S. M., M. R. S. Symp. Proc. 59 (1986) 249.

[28] Forsythe, G. E. and Wasow, W. R., Difference Method for Partial Differential Equations (John Wiley and Sons Inc.) 1960.

[29] Crank, J. and Nicholson, P., Proc. Cambridge Philos. Soc. 43 (1947) 50. 